\title{
LES IMAGES NUMÉRIQUES S'IMAGINENT L'ARCHÄ̈QUE : METTRE EN PERSPECTIVE LES CARTES POSTALES *
}

Moisés de Lemos MARTINS, Madalena OLIVEIRA et Maria da Luz CORREIA **

Résumé : Les cartes postales appartiennent à un temps dans lequel la communication a signifié aussi un échange calligraphique. Elles sont contemporaines de l'image photographique. Les cartes postales sont pour l'histoire de l'écriture épistolaire ce que les weblogs sont pour l'histoire de la cyber-communication. Considérées de différents points de vue comme des moyens de communication marginaux, les cartes postales constituent un univers extrêmement riche de représentations de l'imaginaire contemporain.

Mots clés : carte postale, imaginaire, image, écriture épistolaire, médias.

\begin{abstract}
Picture postcards belong to a time in which communication meant also a type of calligraphic exchange. They are contemporary of photographic image. Postcards are to the history of epistolary writing what weblogs are to the history of cyber-communication. Considered from different points of view as production and marginal means of communication, picture postcards comprise an extremely rich universe of representations of the contemporary imaginary.
\end{abstract}

Keywords : picture postcard, imaginary, image, epistolography, media.

*. Cet article porte sur les cartes postales et s'inscrit dans le cadre d'un projet de recherche collective qui est en développement au Centre d'Études de Communication et Société (CECS), sous le titre "Illustrated Postcards. Towards a socio-semiotics of image and imaginarium ", pouvant être consulté sur le site du CECS, www.cecs.uminho.pt, et dans le blogue http://www.postaisilustrados.blogspot.com/, ce dernier constituant une structure d'appui à ce projet de recherche.

** Centre d'Études de Communication et Société (CECS), Université du Minho, Braga, Portugal. moisesm@ics.uminho.pt ; madalena.oliveira@ics.uminho.pt ; mariadaluzcorreia@gmail.com 
Les cartes postales constituent un vieux medium qui est contemporain de l'invention de l'appareil photo vers le milieu du XIXe siècle. Ce vieux medium, qui n'est pas sans convoquer les réflexions de Walter Benjamin à propos l'âge de la reproductibilité mécanique, est toutefois le précurseur des e-cards, des home pages, des jeux digitaux et des posts en weblogs. Ceci signifie que la carte postale illustrée est, dans une certaine mesure, une façon de traverser la modernité et aussi de comprendre les formes de communication que nous pouvons caractériser comme postmodernes 1 .

Il s'agit d'un phénomène communicationnel en apparence marginal par rapport aux grands médias, d'une part la presse écrite et audiovisuelle, et de l'autre le multimédia et la communication par l'Internet. La carte postale est normalement déconsidérée par rapport à ces grands médias. Mais elle nous permet de comprendre des courants de transformation culturelle, historique et sociologique, d'une époque et d'un endroit précis, au même titre que le font d'autres formats et d'autres supports de la communication. Nous pensons au gramophone, à la caméra à filmer et à la machine à écrire, étudiés par Friedrich Kittler ${ }^{2}$, et pensons de même aux vitrines commerciales. En ce moment où les interfaces numériques font la une, ces supports, en apparence marginaux mais beaucoup plus singularisants que les grands médias, gagnent une importance décisive pour la réappréciation du poids que ceux-ci exercent dans la communauté.

La carte postale valorise, en fait, autant l'interactivité et l'attachement - ce que Michel Maffesoli appelle « l'enracinement dynamique » - que le caractère fragmentaire et multiple de l'existence, l'instantanéité, l'émotion, " l'effervescence de l'instant 3 ». Elle valorise aussi l'hybridation de l'homme et de la machine, mixant la reproduction mécanique des images (aujourd'hui reproduction numérique) et l'expression intime de sentiments. Bref, la carte postale fait une place à l'ordinaire, au banal 4 ; elle valorise le quotidien, "le rythme de la vie ", pour utiliser encore une formule de Maffesoli 5 , faisant l'éloge de la surface qui s'ouvre en profondeur.

1. Cette idée s'articule avec la proposition de Michel Maffesoli, suivant laquelle il y a dans nos sociétés technologiques « le retour à l'antique, à l'archaïque ». Cf. M. Maffesoli, L'instant éternel. Le retour du tragique dans les sociétés postmodernes, Denoël, Paris, 2000.

2. F. Kittler, Gramophone, film, typewriter. Stanford University Press, Stanford, 1999.

3. M. Maffesoli, L'instant éternel. Le retour du tragique dans les sociétés postmodernes. Denoël, Paris, 2000. Bien que la carte postale ne puisse pas être comparée aux dispositifs contemporains de nature électronique, qui mettent en liaison les personnes à une vitesse instantanée, il faut signaler que la rapidité de transmission est le principe même de la carte postale. Selon Tom Phillips, dans quelques villes, au début du XXe siècle, les cartes postales pouvaient être distribuées jusqu'à cinq fois le jour. Pour cette raison, les cartes postales avaient plutôt la fonction attribuée aujourd'hui aux SMS de servir l'envoi de petits messages. T. Phillips, The postcard century, 2000 cards and their messages. Thames and Hudson, Londres, 2000.

4. Cf. Sami-Ali, Le banal, Paris, Gallimard 1980. M. Maffesoli, La Connaissance Ordinaire - Précis de Sociologie Compréhensive. Librairie des Méridiens, Paris, 1985. F. Jost, Le culte du banal. CNRS, Paris, 2007.

5. M. Maffesoli, Le rythme de la vie, La Table Ronde, Paris, 2004. 


\section{Une fenêtre sur le monde}

La carte postale a toujours été liée aux formes les plus intimes de la communication interpersonnelle : elle se destinait assez souvent à la communication à distance, avec la famille et les amis, d'informations non partageables dans les espaces publics ${ }^{6}$. En effet, grâce aux potentialités de l'illustration, la carte postale a contribué à la massification de la correspondance interpersonnelle. Mais la carte postale traditionnelle consiste surtout dans un singulier enregistrement ethnographique des peuples : au recto de la carte postale, il y a la production d'un regard. Il s'agit d'un phénomène culturel, aussi bien qu'un instrument de promotion touristique, un instrument de publicité ou même de propagande politique, qui a accompagné la transformation de la société rurale en société urbaine.

Ce moyen de communication se consolide, surtout, par la fixation d'une mémoire collective, soit produisant un regard spécifique sur un pays, une région et un village donnés, bref produisant l'image qu'une culture donne d'elle-même, soit illustrant et patrimonialisant leurs usages et coutumes. De la même façon que les écrans de cinéma et d'ordinateur, la carte postale constitue une sorte de fenêtre ouverte sur le monde, une fenêtre techniquement orientée, qui détermine les conditions du regard, local, régional et national. Illustrant paysages, monuments, lieux ou figures typiques, les cartes postales sont, plus qu'un album historique d'un peuple, des reflets des figurations humaines qui constituent l'imaginaire contemporain.

Illustration 1 : Reproductions de cartes postales de la première moitié du XXe siècle. Images du nord de Portugal

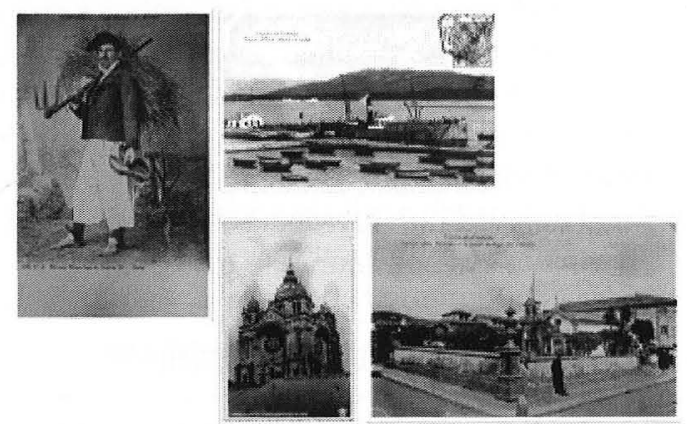

Dans l'image des paysages, bâtiments, représentations politiques, costumes populaires, urbains, ruraux et littoraux, c'est la perception d'un espace qui est dessinée, où la corporéité intercepte ce qu'il y a d'incorporel dans un espace. C'est aussi l'appréhension d'un temps et une suspension de son originalité, de son " ici et maintenant " - c'est l'immobilisation visuelle d'un contexte historique spécifique. Signes d'une sorte de généalogie populaire, les cartes postales enregistrent

6. Voir, à ce propos, H. Joannis-Deberne, Parlez-moi d'amour avec des fautes d'orthographe, Payot, Paris, 2008 ; C. Malaurie, La carte postale, une œuure, Harmattan, Paris, 2003 ; et aussi N. Hossard, Recto-verso. Les faces cachées de la carte postale, Arcadia Éditions, Paris, 2005. 
une compréhension des formes de tradition culturelle et de l'observation de paysages/nature, qui nous permet de comprendre le regard d'une communauté. Ce regard, présent dans les signes visuels, se prête aussi aux réflexions esthétiques : en effet, comme la photographie, les illustrations de ce moyen de communication quotidien comprennent des paramètres de goût et de beau.

Illustration 2 : Reproductions d'une carte postale représentant le travail agricole. Battage du maïs au Minho, Portugal

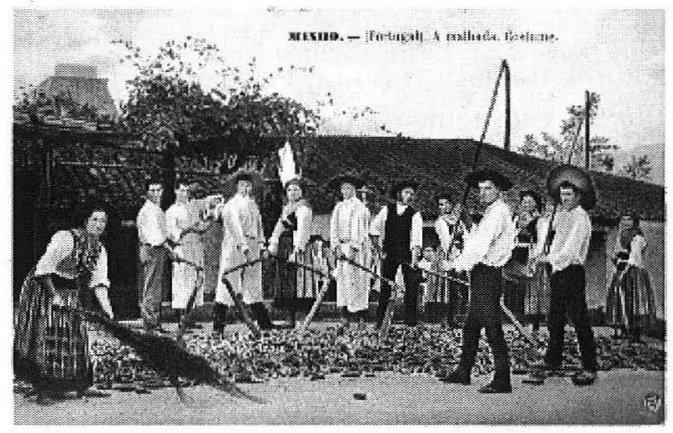

La carte postale renvoie, en effet, à ce qui est explicite dans l'image, par exemple, une forme d'activité plutôt rapportée à la mer, ou alors à la culture de la terre, et de même à un vêtement qui signale une forme de s'habiller. La carte postale valorise ces signes explicites de l'image en tant que représentation. Mais, en même temps, elle renvoie à un implicite subtil qui est à l'œuvre, par exemple, dans la façon de valoriser une pose ou un certain encadrement de l'image, ou alors dans la façon de créer des communautés imaginaires rapportées à l'économie du tourisme ou à des pouvoirs nationaux, régionaux et locaux. À l'époque de la globalisation, la compréhension des cultures locales exige que l'on prête une attention particulière aux mécanismes socio-sémiotiques qui les ont créées, des cultures dont la reconnaissance dépend aujourd'hui du respect du " génie du lieu » et d'une participation équitable à la vie dans la communauté globale.

\section{Une technique de l'image : de l'archive à l'instrumentalisation}

Illustration 3: Reproduction d'une carte postale écrite en 1899

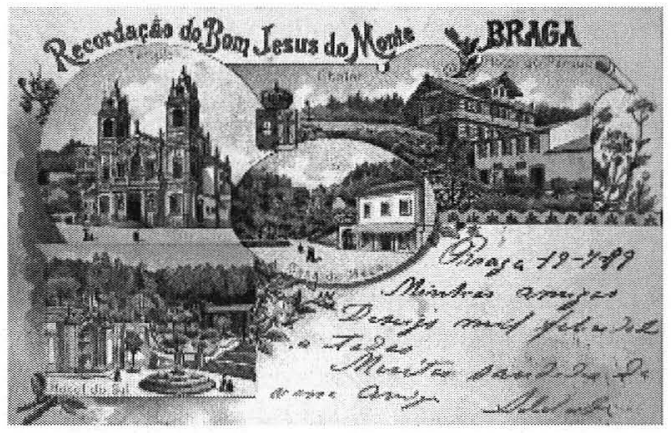


Après les tentatives d'officialisation en 1865 au $5^{e}$ Congrès postal, la première carte postale aurait circulé en Autriche en 1869. Quant à la première carte postale illustrée, il n'existe pas de date reconnue unanimement, mais la plupart des auteurs suggèrent qu'elle est apparue en Allemagne en 1870. La carte postale illustrée eut donc son essor à une époque de vulgarisation de la presse et de grande diffusion des livres illustrés - selon Gervereau 7, une telle période se situe entre 1880 et 1910. En effet, les cartes postales deviennent populaires grâce au désir de circulation d'images qui a accompagné le passage du XIXe au $\mathrm{XX}^{e}$ siècle, ainsi qu'en raison de leur aspect économique : la carte postale était un objet de consommation accessible et son envoi était plus économique que l'envoi d'une lettre. Les deux premières décennies du XXe siècle, considérées comme l'âge d'or de ce moyen de communication ${ }^{8}$, furent une période glorieuse pour l'histoire de la carte postale. Le tournant du XXe siècle fut marqué par des progrès techniques dans les arts graphiques, spécialement en ce qui concerne les techniques d'impression des images. À la Belle Époque, les éditions de cartes postales se multipliaient dans tous les pays à un rythme qui promettait d'assurer une documentation effective de l'histoire, des coutumes et de l'autoreprésentation des peuples. Apparue à une époque où la photographie avait déjà conquis du territoire par rapport à la peinture, la carte postale est reproduite en masse par différentes techniques d'impression (photographie, phototypie, typographie...). En tant que support de reproduction, la carte postale évoque ainsi le déferlement des images.

Bien souvent, notre époque est définie comme une civilisation de l'image 9. L'image n'est cependant pas le signe de l'originalité de notre temps. Ce qui caractérise notre période historique et la singularise dans l'histoire, c'est bien la production industrielle des images et leur reproduction technologique, qui a débuté avec l'avènement de la photographie. La technique abandonne désormais l'iconoclastie au profit de l'iconophilie ${ }^{10}$. C'est avec la photographie, vers la moitié du XIXe siècle, que l'image prend définitivement le chemin de la séparation de la parole et devient autotélique, séparée 11 . Image fixe, l'illustration de la carte postale ne possède pas le mouvement des images du cinéma et elle se distancie encore plus des ambiances numériques, mais, en tant qu'image technique, elle participe déjà à la multiplication autonome des images, propre à «l'âge de la reproductibilité mécanique 12 ".

Dans une histoire visuelle du XXe siècle, Laurent Gervereau 13 signale que notre temps se caractérise par « l'accumulation d'images sur tout support, de toutes

7. L. Gervereau, Histoire du visuel au XXe siècle, Éditions du Seuil, Paris, 2003.

8. Malaurie et Hossard classifient la période de 1900 à 1920 comme le premier âge d'or de la carte postale. Cf. C. Malaurie, op. cit. ; N. Hossard, op. cit.

9. Cf. M. L. Martins, A linguagem a verdade e o poder. Ensaio de Semiótica Social. Fundação Calouste Gulbenkian/Fundação para a Ciência e a Tecnologia, Lisboa, 2002.

M. L. Martins, "Ce que peuvent les images. Trajet de l'un au multiple", in Les Cahiers Européens de l'Imaginaire, n¹, CNRS, Paris, pp. 158-162, 2009.

10. M. Maffesoli, Au Creux des Apparences. Pour une éthique de l'esthétique, Plon, Paris, 1990, p. 111.

11. M. L. Martins, op. cit.

12. W. Benjamin, Sobre arte, técnica, linguagem e política, Relógio d'Água, Lisboa, 1992.

13. L. Gerverau, op. cit., p. 30. 
les époques, de toutes les civilisations ». Gervereau reprend ainsi le thème de l'archive, un thème que nous voyons développer depuis Foucault 14 et qui est particulièrement approfondi par Derrida 15 et Baudrillard. Derrida signale d'ailleurs que nous sommes en "mal d'archive ", une fièvre qui nous embrase : nous sommes compulsifs de cette course qui révèle le désir nostalgique et répétitif des retours, de la restauration et de la récupération 16 . Baudrillard ne dira pas autre chose : nous sommes engagés dans une tâche de constant rebobinage du passé, une tendance à « tout historiciser, tout archiver, tout mémoriser sur notre passé et sur celui de toutes les cultures 17 ». Persécuté par le fantasme de l'oubli, notre temps est assiégé par les techniques d'archive de toutes sortes, lesquelles sont de plus en plus complexes, raffinées, nombreuses et puissantes.

En effet, la carte postale est synonyme d'image abondante ainsi que d'une impulsion généralisée de mémorisation. Comme l'a dit Tom Phillips, " peu de choses, de personnes ou de lieux n'ont pas fini à un moment ou un autre comme un thème, ou une partie involontaire ou non intentionnelle d'une carte postale 18 ». En ce qui concerne les thèmes, les cartes postales ont toujours fait preuve d'une diversité surprenante... Elles documentent paysages, monuments, œuvres d'art, illustrations humoristiques, elles signalent dates et événements importants. À l'époque des Iphones, la carte postale est toujours un outil du marketing touristique, se prêtant en même temps à servir de souvenir personnel d'un voyage. L'édition de cartes postales à l'occasion d'événements marquants comme les expositions universelles de la fin du XIXe et du début du XXe siècle, mais encore aujourd'hui à la suite d'inaugurations de monuments et de commémorations, témoigne de la façon dont ce moyen de communication répond aux besoins d'« historicisation ».

Illustration 4 : Reproductions de cartes postales de la période de l'instauration de la république au Portugal, en 1910
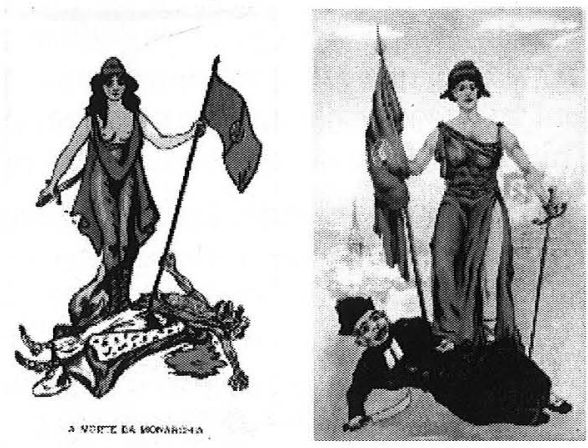

14. M. Foucault, Les mots et les choses. Une archéologie des Sciences Humaines, Gallimard, Paris, 1966.

15. J. Derrida, Mal d'archive, Ed. Galilée, Paris, 1995. J. Baudrillard, A greve dos acontecimentos, Teorema, Lisboa, 1992.

16. J. Derrida, op. cit, p. 142.

17. J. Baudrillard, op. cit., p. 19.

18. T. Phillips, op. cit., p. 17. 
En outre, dans la première moitié du XXe siècle, suivant ce qui est arrivé aux arts visuels en général (la photographie, le cinéma, la télévision), les cartes postales et leurs représentations furent appropriées par les États et instrumentalisées au profit de la propagande politique. Le domaine publicitaire rencontre aussi dans les cartes postales un outil pour la diffusion de messages commerciaux. La publicité illustrée fut, indubitablement, une des plus importantes découvertes de la fin du XIXe siècle. Prospère en termes industriels et commerciaux, le premier quart du XXe siècle fut en général favorable au développement de l'activité publicitaire, qui a trouvé dans les cartes postales un de ses supports les plus lucratifs. Aujourd'hui, la carte postale, publicitaire et touristique, est toujours un outil de diffusion de messages commerciaux. Étant donné que la carte postale est actuellement éditée et produite par de puissantes « industries de séduction 19 ", qui mélangent l'histoire et l'art avec le tourisme et les loisirs, et mettent l' "équipement esthétique " au profit de la "rationalité technique ", on peut toujours mettre en question l'instrumentalisation de ce support visuel. L'idée d'une « disneylandisation du monde » contemporain, qui serait accomplie par la carte postale, telle qu'elle est énoncée par Nicolas Hossard ${ }^{20}$, n'est pas loin de notre point de vue.

Illustration 5 : Reproductions de cartes postales publicitaires.

A la gauche, une annonce à l' huile d'olive, et à droite une image publicitaire des automobiles Peugeot

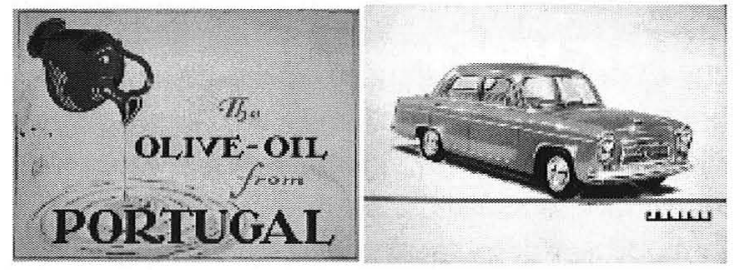

\section{La carte postale à l'ère du Web 2.0}

En tant que précurseur des cartes postales électroniques (e-cards), des home pages, des jeux digitaux et des posts en weblogs, la carte postale appartient, comme nous l'avons signalé, à l'histoire de la communication, qui comprend l'histoire des technologies de l'information par l'image. Entre l'image gravée, en termes rudimentaires, sur papier, et l'image de nature numérique, plus d'un siècle s'est écoulé et, de même, une transformation profonde des modes de communication s'est vérifiée. La carte postale n'a pas, en effet, ce rythme " quasi instantané » que Jacques Derrida 21 assignait à un format révolutionnaire tel que l'e-mail. L'expérience de communication à l'œuvre dans une carte postale traditionnelle suppose la constitution

19. M. L. Martins, A linguagem a verdade e o poder. Ensaio de Semiótica Social, Fundação Calouste Gulbenkian/Fundação para a Ciência e a Tecnologia, Lisboa, 2002, p. 184. 20. N. Hossard, op. cit., p. 84.

21. J. Derrida, op. cit., p. 36. 
d'espaces et de temps différents, et aussi une traversée du temps et de l'espace, qui mettent en connexion l'expéditeur et le destinataire et en même temps les séparent. À une époque marquée par « une espèce de primitivisme ultra-tech, où tout est en train de devenir connecté : les choses, les images, les objets, les corps et les machines ", avec l'abolition de "la distance entre liaison et dé-liaison », la carte postale permet encore de nous connecter et de nous déconnecter, elle sépare et rassemble, tout en respectant la " nature divisée et divisible de l'humain 22 ». En effet, l'acte d'envoyer ou de recevoir une carte postale nous impose nécessairement de parcourir une distance de temps et d'espace, qu'elle soit petite ou grande, entre celui qui envoie et celui qui reçoit. Contrairement aux moyens de communication électroniques, toute carte postale est nécessairement associée à un espace physique concret, à un expéditeur et à un destinataire inscrits sur une adresse réelle.

C'est certainement en prenant en compte ces aspects que, dans les années 1980, Jacques Derrida prévoyait une fin proche de ce moyen de communication 23. Il se peut effectivement qu'à l'âge de "l'extase de la communication", pour reprendre une idée de Jean Baudrillard ${ }^{24}$, les cartes postales traditionnelles aient perdu l'élan qu'elles ont connu du milieu du XIXe siècle aux premières décades du $\mathrm{XX}$. Il est bien probable qu'elles exercent de moins en moins la fonction de moyen de communication interpersonnelle et affective. Cependant, plusieurs auteurs décrivent l'histoire de la carte postale d'une façon qui contredit en grande partie une vision apocalyptique de son trajet. Malaurie, ainsi qu'Hossard 25, considèrent que de 1975 à nos jours, nous vivons le deuxième âge d'or de ce morceau de papier cartonné. Les articles de la presse internationale parus récemment sur ce sujet 26 , en révélant certaines données statistiques sur la production, la consommation et l'utilisation des cartes postales dans l'actualité, confirment ce renouveau de la carte postale.

22. J. B. Miranda, Crítica das Ligações na Era da Técnica, Tropismos, Lisboa, 2001, pp. 269-270.

23. J. Derrida, La carte postale de Socrate à Freud et au-delà, Flammarion, Paris, 1980.

24. J. Baudrillard, L'autre par lui-même, Ed. Galilée, Paris, 1987.

25. C. Malaurie, op. cit. ; N. Hossard, op. cit.

26. Le 17 juillet 2008, le Travel Blog - The Guardian signalait, dans un post, dont le titre fut "Postcards back from the edge", la resurgence de la fortune des cartes postales. L'éditeur pense deux choses à ce propos: d'une part, qu'il est possible d'imaginer que cette nouvelle vie de la carte postale n'est pas seulement l'effet de la résurgence des vacances au bord de la mer en Grande Bretagne, mais qu'elle la promeut aussi ; d'autre part, cette redécouverte des cartes postales peut promouvoir la marche à pied, dans le forme d'une grande promenade vers les derniers post office qui restent. 
Illustration 6 : Carte Postale publiée par les Éditions Maurice Juan

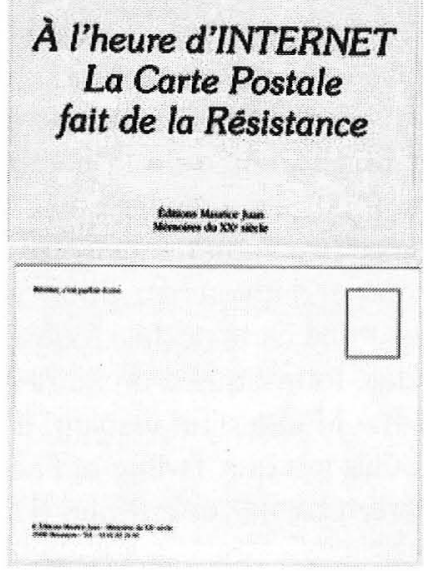

Une des raisons de ce renouveau peut être la multiplicité des fonctions de ce moyen de communication. Bjarne Rogan 27 se rapporte aux différents usages de ce support, en parlant des «fonctions imbriquées » de la carte postale. Elle explique que la carte postale peut être analysée comme " un objet de collection, un cadeau, un souvenir, un moyen de communication... ". Nicolas Hossard 28 , pour sa part, décrit aussi la "place originale » de la photographie de la carte postale " dans l'univers des images » : au-delà de sa fonction de correspondance, les illustrations de la carte postale peuvent servir des buts publicitaires, artistiques, mémoratifs et documentaires. En effet, les cartes ne sont pas des objets fonctionnels comme n'importe quel objet : elles ne sont pas comme le télégramme et encore moins le minitel. La carte postale est un outil de correspondance avec image, elle est un objet esthétique, voire un « objet mythologique » - au sens où Baudrillard 29 utilise cette expression.

Illustration 7 : Logo de Postcrossing

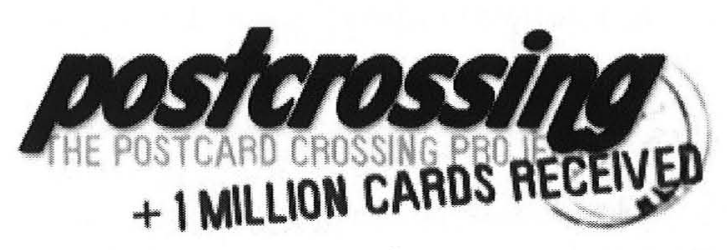

27. B. Rogan, An entangled object: The Picture Postcard as Souvenir and Collectible, Exchange and Ritual Communication. (http://socrates.berkeley.edu/ caforum/volume4/ vol4_article1.html).

28. $\bar{N}$. Hossard, op. cit., p. 38.

29. J. Baudrillard, Le système des objets, Éditions Gallimard, Paris, 1968. 
Objet de collection à la mode, la carte postale est aujourd'hui stockée dans de puissantes data bases comme Ebay qui réactivent online, un peu partout dans le monde, la traditionnelle activité de la cartophilie. Redevenue populaire parce que de plus en plus rare, la correspondance par la carte postale est aujourd'hui au centre d'une communauté virtuelle comme Postcrossing, qui l'utilise comme moyen d'échange. Ce réseau, fondé en 2005 et qui comprend 97027 membres d'environ 200 pays différents, est un bon exemple de la combinaison de la pratique de l'envoi traditionnel de la carte postale avec les outils technologiques contemporains. En accédant au site de cette communauté internationale, on peut s'inscrire, demander une adresse de courrier d'un autre membre (choisi au hasard par le système), envoyer à cette adresse une carte postale touristique par la poste, attendre la réception d'une carte postale touristique d'un autre membre et puis enregistrer la carte reçue dans le système. Munie d'un weblog, d'un forum, d'une page de Flickr, en utilisant d'autres outils tels que Twitter et Facebook, cette communauté montre bien comment la carte postale est en train d'être assimilée aux réseaux sociaux numériques.

Illustration 8 : Reproduction du recto d'un freecard

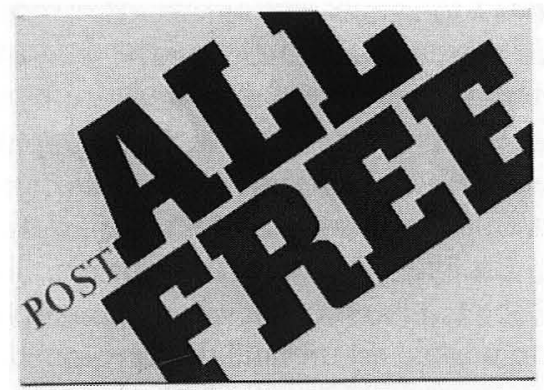

Finalement, l'avènement du numérique a certainement facilité la production et la reproduction de ce support touristique, dans son format traditionnel. La carte postale illustrée contemporaine continue à être produite, en employant maintenant des processus technologiques qui simplifient l'édition. La carte postale se maintient ainsi dans ses formats les plus classiques (les cartes de prises de vues typiques, les cartes de vœux stéréotypés...), et elle se renouvelle aussi avec l'avènement des freecards (cartes postales publicitaires gratuites).

L'apparition des cartes postales publicitaires gratuites, dont les vitrines sont $\mathrm{si}$ présentes dans notre quotidien (avec les flyers, mupies, billboards), et tout le carnaval publicitaire qui envahit nos espaces publics et privés, est bien un indice que la carte postale constitue un format de communication qui reste à encadrer dans notre contexte communicatif technologique. Nous nous rendons compte aussi que les cartes postales qui reproduisent des œuvres d'art, dans des musées et des librairies, constituent un commerce assez lucratif dans le domaine du tourisme. 
Illustration 9 : Page d'accueil du site e-card.com

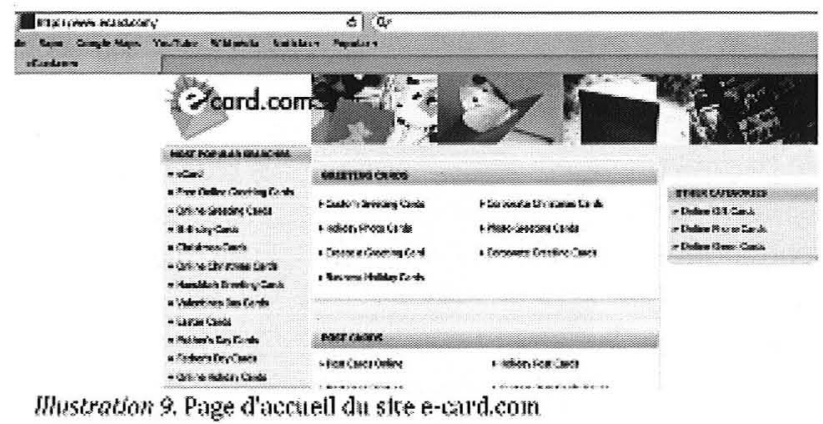

Finalement, il est nécessaire de considérer les manifestations qui intègrent la vieille carte postale à de nouveaux artefacts digitalisés, des artefacts hybrides, de son, lumière, couleur et sensibilité, tels que les e-cards, de même que les posts dans les weblogs, les MMS et des D-cartes. À notre avis, la récupération de la carte postale par le cyberespace sous la forme de l'e-card, son remplacement par le format des SMS (Short Messages Service), MMS (Multimedia Messaging Service) dans les portables, ou encore leur transformation dans un format multimédia tel que les Dcartes (des cartes postales avec un DVD, y compris des photos ou des films), signalent avec évidence le rajeunissement de ce moyen traditionnel de communication. Enfin, la récente application Postman de Iphone est aussi illustrative de nos propos : cette fonctionnalité qui se sert de la technologie de la géolocalisation permet de créer des cartes postales électroniques par lesquelles, au-delà d'écrire un message et de choisir une photo, on montre notre localisation géographique sur une carte. Ces cartes postales électroniques peuvent ensuite être envoyées par téléphone ou diffusés dans des réseaux sociaux comme Twitter ou Facebook...

Certes, la médiation est devenue presque immédiate dans les cartes postales numériques. Mais celles-ci gardent, quand même, les caractéristiques principales des moyens de communication traditionnels, qui ont joué un rôle si important dans la construction de l'imaginaire populaire, notamment une écriture légère et rapide, ancrée dans des images illustratives - ethnographiques, géographiques, historiques, patrimoniales - ou humoristiques, satiriques et publicitaires.

La carte postale permet ainsi de tracer le chemin entre les formes interpersonnelles de communiquer de l'âge de la poste et de la reproductibilité analogique, et les supports de l'époque du Web 2.0 et du numérique. À ce propos, une récente étude qui entreprend une comparaison entre la carte postale du début du XXe siècle et les micro-posts de Twitter, réalisée à l'Université de Lancaster et à l'Université Métropolitaine de Manchester (Royaume-Uni), sous la direction de Julia Gillen et Nigel Hall, est fort illustratif. Ces chercheurs, qui envisagent la carte postale edwardienne en tant que technologie de communication multimodale, expriment un point 
de vue semblable au nôtre, lorsqu'ils soulignent que la carte postale est le premier support à permettre d'exercer une écriture quotidienne et informelle 30 .

\section{Un dernier mot}

La carte postale permet un va-et-vient des formes les plus traditionnelles de transmission de messages à des formes totalement numériques. Ce chemin, auquel nous sommes convoqués par la carte postale, permet de mieux comprendre le rôle joué par la technologie dans la définition de l'humain. Moyen de correspondance informelle et fragmentaire, la carte postale est un précurseur des formes de communication dites postmodernes et devient un objet propice à l'étude du quotidien, dans son rapport avec l'imaginaire.

Si le quotidien s'oppose à l'imaginaire, comme le pense Sami-Ali 31 , la carte postale semble un objet où ces deux dimensions de la vie - l'ordinaire et l'onirique - ne cessent de se croiser. Quand on écrit une carte postale ou quand on envoie un MMS, on ne fait que reprendre les " bons mots » et " salutations qui ponctuent les trajets quotidiens ", mentionnés par Michel Maffesoli 32. De manière électronique comme sur le papier illustré de la carte postale, c'est l'instant transcrit - gardé ou oublié - qui compte. Avec sa brève littérature épistolaire, la carte postale, apparue à la même époque que la photographie, éprouve le même goût que cette dernière pour l'instantané. La carte postale illustrée, c'est un geste qui, dans sa trivialité, signifie surtout ceci : “ J'ai été là et j'ai pensé à toi. » Le message et l'image analogiques ou numériques sont souvent redondants et puisent dans les lieux communs du répertoire expressif populaire : ce sont des clichés et des formules toutes faites... En ce sens, la carte postale rejoint l'esthétique du « banal » telle qu'elle est comprise par Sami Ali : il s'agit du rythme répétitif et cyclique de nos vies - le monotone, l'uniforme, l'indifférent.

30. Selon cette étude, la carte postale est un précurseur des messages de Twitter, à cause de différents aspects : les posts de ce réseau social sont limités à 140 caractères ; semblablement, le message de la carte postale est restreint par la dimension réduite de son verso ; les deux supports suscitent des attentats à la grammaire ; finalement, le twitter, qui obtient 55 millions de visites par mois, est aussi populaire que les cartes postales à leur âge d'or en Angleterre.

31. Sami-Ali, Le banal, Gallimard, Paris, 1980.

32. M. Maffesoli, La conquête du présent. Pour une sociologie de la vie quotidienne, Desclée de Brower, Paris, 1998, p. 91. 
Illustration 10 : Carte postale d'amoureux, reproduite sur le site http://postais.do.sapo.pt/

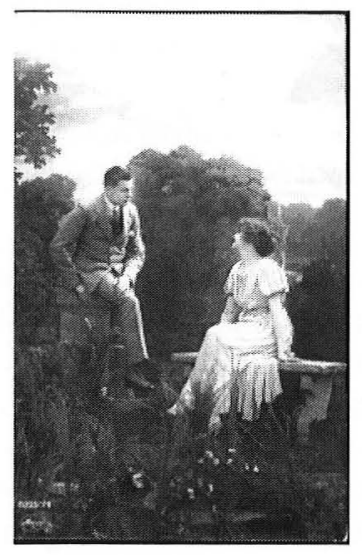

Cependant, comme le mentionne encore Sami-Ali 33, les objets ordinaires convoquent une "activité fantasmatique » et un imaginaire qui s'opposent constamment à leur banalisation. La carte postale ne fait pas exception. Par exemple, comme l'a montré Henri Joannis-Deberne à propos de la poésie stéréotypée des cartes postales d'amour - ou des SMS d'amour -, la forme, et très spécialement les transgressions de la forme comme les fautes d'orthographe, donnent lieu à l'authenticité. Ce support, tout en conservant une certaine légèreté - conférée par l'aspect standardisé des images et des messages -, met l'accent sur le rapport émotif et intersubjectif. La carte postale accomplit ainsi une synthèse entre un détachement insouciant et une proximité «sensologique » pour gloser Mario Perniola 34. Une telle synthèse est bien explicite dans un commentaire publié au début du siècle dans Ilustração Portuguesa : "Elle [la carte postale] possède le charme de toutes les tendresses sans conséquences et de tous les petits mensonges sans compromis. $\mathrm{Ni}$ la sécheresse mercenaire du télégramme, ni l'intimité ennuyeuse, grave, périlleuse, des deux feuilles de papier à lettre. »

La sensibilité et l'émotivité de ce moyen de communication suggèrent une esthétique de la vie qui s'exprime dans la légèreté du support destiné à peu de mots et dans l'important échange émotif qui l'enveloppe. À une époque où c'est l'" ambivalence » qui caractérise fondamentalement les rapports humains ${ }^{35}$, c'est aussi le dialogue affectif, présent autant dans la carte postale que dans les messages électroniques, qui confère la richesse polysémique à un message apparemment stéréotypé et qui se prête au cliché de la multiplicité des regards. En outre, la pratique de correspondance par carte postale est un rituel associé aux loisirs et aux voyages, et

33. Sami-Ali, op. cit., p.23.

34. M. Perniola, Do Sentir, Presença, Lisboa, 1993.

35. M. L. Martins, "A mobilização infinita numa sociedade de meios sem fins", in Álvares, Cláudia \& Damásio, Manuel (Org.) Teorias e práticas dos media. Situando o local no global. Edições Lusófonas, Lisboa, 2010. 
son message accompagne souvent des cadeaux. De cette sorte, la carte postale convoque sûrement l'autre, mais en outre elle convoque des occasions de liberté et d'agitation ainsi qu'une grande charge imaginale, au même titre que les voyages et les rassemblements festifs... Dans ce sens, si elle est un registre des peuples, de leur tradition et de leur identité, elle est aussi associée à des expériences de nomadisme et de multiplicité, s'ajustant à l'idée d' " enracinement dynamique » développée par Michel Maffesoli.

Média marginal, la carte postale s'accorde surtout avec la sensibilité postmoderne, où « le présent, les circonstances, l'aléatoire et les émotions 36 " gagnent de plus en plus d'importance. Images toutes faites, les cartes postales sont souvent synonymes du cliché, de la représentation passe-partout, du message laconique et peu imaginatif. Mais c'est tout au long de ces surfaces profondes ${ }^{37}$ que se décide un échange ; le geste très concret de partager une image, autant par la poste que par le téléphone portable, n'est-il pas souvent chargé de poésie ? Certes, la rencontre humaine supposée par la carte postale intégrait la calligraphie et la texture du papier, tandis que les écrans ne nous donnent aujourd'hui qu'une écriture dépersonnalisée et froide. Toutefois, les cartes postales, ainsi que leurs héritiers numériques, ont bien ceci en commun : ce sont deux objets du quotidien et deux dispositifs de communication singularisante qui façonnent l'humain. En nous référant à ces formes de communication analogiques et numériques, nous pouvons dire que l'humain possède désormais une peau technicienne ${ }^{38}$, bien qu'il ne cesse de revenir en même temps à ce qu'il y a de plus archaïque chez lui : l'émotion, le rêve, le mythe...

\section{Bibiographie}

Baudrillard J., A greve dos acontecimentos. Lisboa, Teorema, 1992

Baudrillard J., L'autre par lui-même. Paris, Galilée, 1987

Baudrillard J., Le système des objets. Paris, Gallimard, 1968

Benjamin W., Sobre arte, técnica, linguagem e política. Lisboa, Relógio d'Água, 1992

Birnbaum A., "Transmissions d'images : éloge de la carte postale ", in École des Arts Décoratifs de Strasbourg, Tradition, transmission, enseignement. Une relecture de la modernité par Walter Benjamin. Strasbourg, École des Arts Décoratifs, 1997.

Derrida J., La carte postale de Socrate à Freud et au-delà. Paris, Flammarion, 1980.

Derrida J., Mal d'archive. Paris, Galilée, 1995.

Foucault M., Les mots et les choses. Une archéologie des sciences humaines. Paris, Gallimard, 1966.

\section{Ibidem.}

37. La surface est ici comprise par nous comme le "creux des apparences" mentionné par Michel Maffesoli, c'est-à-dire, comme une "profondeur de ce qui est superficiel", pour reprendre les termes de Maurice Blanchot. En effet, si la carte postale avec sa réduite et anodine surface donne lieu à un échange intime, d'une façon plus générale, c'est aussi "à la surface que l'humain s'ouvre en profondeur" (M.L. Martins, op. cit.).

38. D. De Kerckhove, A pele da cultura - uma investigação sobre a nova realidade electrónica, Relógio d'Água, Lisboa, 1997. 
Gervereau L., Histoire du visuel au XXe siècle. Paris, Seuil, 2003.

Hossard N., Recto-verso. Les faces cachées de la carte postale. Paris, Arcadia, 2005.

Joannis-Deberne H., Parlez-moi d'amour avec des fautes d'orthographe. Paris, Payot, 2008.

Jost F. Le culte du banal. Paris, CNRS, 2007.

Kerckhove D. de, A pele da cultura : uma investigação sobre a nova realidade electrónica. Lisboas, Relógio d'Água, 1997.

Kittler F., Gramophone, film, typewriter. Stanford, Stanford University Press, 1999.

Maffesoli M., Le rythme de la vie. Paris, La Table Ronde, 2004.

Maffesoli M., L'instant éternel. Le retour du tragique dans les sociétés postmodernes. Paris, Denoël, 2000.

Maffesoli M., La conquête du présent. Pour une sociologie de la vie quotidienne. Paris, Desclée de Brouwer, 1998.

Maffesoli M., Au creux des apparences. Pour une éthique de l'esthétique. Paris, Plon, 1990.

Maffesoli M., La connaissance ordinaire. Précis de sociologie compréhensive. Paris, Librairie des Méridiens, 1985.

Malaurie C., La carte postale, une ceuvre. Paris, L'Harmattan, 2003.

Martins M. L., A linguagem a verdade e o poder. Ensaio de Semiótica Social. Lisboa, Fundação Calouste Gulbenkian/Fundação para a Ciência e a Tecnologia, 2002.

Martins M. L., "Ce que peuvent les images. Trajet de l'un au multiple ", Les Cahiers Européens de l'Imaginaire, $\mathrm{n}^{\circ}$ 1, 2009, pp. 158-162.

Martins M. L., "A mobilização infinita numa sociedade de meios sem fins", in Cláudia Álvares et Manuel Damásio (Eds.), Teorias e práticas dos media. Situando o local no global. Lisboa, Edições Lusófonas, 2010.

Miranda J. B. (Ed.), Crítica das Ligações na Era da Técnica. Lisboa, Tropismos, 2001.

Perniola M., Do Sentir. Lisboa, Presença, 1993.

Phillips T., The postcard century, 2000 cards and their messages. London, Thames and Hudson, 2000.

Rogan B. (2005), "An entangled object: The Picture Postcard as Souvenir and Collectible, Exchange and Ritual Communication". En ligne sur http://socrates.berkeley.edu/ caforum/volume4/vol4_article1.html.

Sami-Ali Le banal. Paris, Gallimard, 1980.

\section{Autres références}

Ilustração Portuguesa, Ile Série, n 572, 5 février 1917, p. 104.

"Postcards back from the edge », Travel Blog - The Guardian (July 17, 2008), http:// www.guardian.co.uk/travel/blog/2008/jul/17/postcardsbackfromtheedge (consulté le 7 juin 2010).

«The Edwardian Postcard », http://www.Lancs.ac.uk/fss/centres/llrc/activities/641 (consulté le 27 juin 2010). 